\title{
Anesthesia For Laparoscopic Cholecystectomy In A Patient With Transverse Myelitis: A Case Report
}

\author{
Achyut Sharma ${ }^{* 1}$, Shikshya Khatiwoda ${ }^{2}$, Kishor Khanal ${ }^{2}$, Apurb Sharma' ${ }^{2}$, Prajjwal Raj Bhattarai ${ }^{2}$ \\ 'Specialty registrar, Intensive Care Unit, Harefield Hospital, Royal Brompton and Harefield NHS Trust (Part of Guy's and St. Thomas' \\ Trust), London, UK
}

${ }^{2}$ Department of Anesthesia, Pain Management, and Critical Care, Nepal Mediciti Hospital, Sainbu, Lalitpur, Nepal

$\begin{array}{lll}\text { BASTROUND } & \text { ABSTR } & \begin{array}{l}\text { This work is licensed under a Creative } \\ \text { Commons Attribution 4.0 Unported License. }\end{array}\end{array}$

Transverse Myelitis is a rare inflammatory disorder of the spinal cord presenting with sensory, motor, and autonomic dysfunction. Patients of transverse myelitis may present to operation theater for a variety of surgical procedures. The debilitating nature of the disease and interaction with anesthetic agents poses serious challenges during perioperative period. Choice of anesthesia is debatable but the complication following anesthesia can be prevented with thorough preoperative preparation and judicious use of anesthetic agent with neuromuscular monitoring. We present a successful conduction of laparoscopic cholecystectomy in a patient with transverse myelitis.

\section{CASE PRESENTATION}

A 55-year-old female who was a known case of transverse myelitis presented for laparoscopic cholecystectomy to remove a gall bladder polyp. Except for weakness in bilateral lower limbs which had improved since her diagnosis of transverse myelitis two months back, she had no significant past medical and surgical history. Considering the grave possibility of malignant potential in polyp, cholecystectomy was urgent. Her vitals were within normal limits. Other systemic examinations revealed no abnormalities. She was planned under general anesthesia with avoidance of muscle relaxants and intraoperative use of bispectral index and train-of-four monitoring. The intra-operative period was successfully managed with propofol induction, fentanyl as analgesia, I-gel for maintenance of airway, and isoflurane for maintenance of anesthesia. Bispectral index was targeted to 40-60. Intra-operative, and post-operative period was uneventful and she had no exacerbation in her symptoms in the subsequent follow-up.

\section{CONCLUSION}

As transverse myelitis is an uncommon condition and often not encountered routinely, the patient present real anesthetic challenge for an unsuspecting anesthesiologist. The successful management of such case especially with the use of novel anesthetic monitoring technique provide us with sufficient evidence that dreadful complications of transverse myelitis like exacerbation of motor, sensory and autonomic dysfunction can be avoided.

\section{KEY WORDS}

Anesthetic technique, Transverse Myelitis, TOF ratio, BIS, neuromuscular blockers

\section{BACKGROUND}

Transverse Myelitis (TM) is an acquired disorder of the spinal cord presenting with rapid development of sensory impairment of varying degree, motor weakness, and occasionally associated with bowel and bladder dysfunction. The etiology of TM is variable with causes ranging from post infectious complication to an association with multitude of other neuro-inflammatory disorders like encephalitis, multiple sclerosis, etc.' Although the disease itself is rare, it is not uncommon for affected people to undergo a variety of operative procedures which may include emergency and routine procedures. Conventionally trained anesthesiologists face a real challenge in the management of such cases. An availability and expertise in management of different novel techniques like bispectral index (BIS) and train-of-four (TOF) monitoring help avoid serious consequences. We present a successful management of a laparoscopic cholecystectomy in a person with TM.

\section{CASE PRESENTATION}

A 55-year-old female diagnosed with TM along with Gall bladder polyp was posted for laparoscopic cholecystectomy. At the first presentation 2 months prior to

\section{*Corresponding Author}

Dr. Achyut Sharma

Specialty registrar, Intensive Care Unit, Harefield Hospital, Royal Brompton and Harefield NHS Trust (Part of Guy's and St. Thomas' Trust), London, UK.

Email: achyutsharma@gmail.com | Phone Number: (+44) 07415992798 
surgery, her symptoms were fever, vomiting, and ascending weakness with loss of motor function over the period of 6 days. The patient had constipation with difficulty in passing urine. She did not have any similar illness in the family. Past medical history of the patient was insignificant and had no known drug allergy. On examination, vitals were normal with no evidence of autonomic dysreflexia. Neurological assessment revealed power of 2/5 bilaterally in the lower limb with normal tone. Neurological examination of the upper limb was normal. Fig. 1 A magnetic resonance imaging (MRI) of brain and spinal cord done then revealed swelling in the posterior spinal cord from L2 to L5 which was considered as an inflamed area. Fig. 2

Routine blood works including liver function tests, and thyroid function tests were within normal limits. MRI of spine showed findings suggestive of transverse myelitis from $L 2$ to $L 5$ region which was further corroborated in routine CSF examination. Immediate pre-operative assessment revealed an improved motor power of 3/5 in both the lower limbs and a relatively improved bowel and bladder function. Although the anesthetic concerns and perioperative complications in a case of TM are often serious, a cholecystectomy was necessary as there was a suspicion of a mass (polyp) in the gall bladder.

Anesthetic plan of general anesthesia without muscle relaxant was made as it was deemed necessary to avoid relaxation to prevent exacerbation of symptoms of transverse myelitis. Patient and patient's relatives were explained thoroughly regarding the plan and probable perioperative complications.

In the operating room, standard monitors were attached along with arterial line, and BIS. Patient was induced with propofol $2 \mathrm{mg} / \mathrm{kg}$; fentanyl $2 \mathrm{mcg} / \mathrm{kg}$ was used for analgesia and l-gel size 3 was used to secure airway. Immediate decompression of the stomach was done with a suction catheter to facilitate the surgery and minimize respiratory distress. Isoflurane titrated to minimum alveolar concentration (MAC) of 1.2 was used for maintenance along with oxygen, and BIS was targeted between 40-60 for the adequacy of depth of anesthesia. Positive pressure ventilation was used and train of four (TOF), and end tidal CO2 was monitored continuously (Fig 3). The intraoperative period was uneventful and the total surgical duration was 45 minutes. Towards the end of the surgery, anesthetic gases were discontinued and after the patient had full recovery of all the protective reflexes and a TOF ratio of 0.9, I-gel was removed. Immediate neurological reassessment to evaluate motor and sensory function was performed which was unchanged from pre-operative assessment. In the immediate post-operative period, neurological examination was performed on regular basis for the next 24 hours. The post-operative period and ward stay was uneventful and patient was discharged to home after 3 days. A follow up two weeks later in OPD was normal with no significant neuromuscular problems and the biopsy report was negative for malignancy.

\section{DISCUSSION}

Transverse myelitis is an acquired inflammatory disorder of the spinal cord in which varying segments are affected with wide range of sensory, motor, and autonomic symptoms. When such patients present for surgeries, anesthesiologists are posed with several challenges. The pertinent issues in terms of anesthetic management include the relative contraindication of neuraxial anesthesia, and almost invariable necessity of general anesthesia. Even with general anesthesia, there is an immediate need of avoidance of muscle relaxation in order to mitigate the chances of flaring up of existing weakness.

Cases have been reported on the prolonged effect of rocuronium in the patients with TM2paraplegia following sole epidural anesthesia ${ }^{3}$, transverse myelitis after spinal ${ }^{4}$ or combined spinal epidural analgesia (CSEA) ${ }^{5}$, paralysis following epidural steroid injection ${ }^{6}$. Prolonged effect up to 3 hours 15 minutes of rocuronium had to be reversed by sugammadex. Factors contributing paraplegia after epidural anesthesia could be due to spinal cord infarction (SCI), intramedullary tuberculoma or neoplasm. SCI could cause systemic hypotension, compression effect of local anesthesia possibly leading to thrombosis of the spinal arteries $^{2}$. Similar complication leading to occurrence of TM has been reported by Seok et al. ${ }^{5}$ So the use of regional anesthesia as well as muscle relaxant is still debatable which is contrary from our case to the case reported above. The differences in our case report is avoidance of muscle relaxant or regional anesthesia and the use of advanced monitoring like BIS and TOF ratio which was not monitored in the cases reported by $\mathrm{P}$ Walsh ${ }^{7}$ and $\mathrm{G}$ Weekes et $\mathrm{al}^{2}$. Case reported by Balakrishnan ${ }^{8}$ is similar to our case which was done without using muscle relaxants but the TOF ratio and BIS monitoring was not done. So the use of TOF ratio not only guides in managing the depth of anesthesia but also prevents the incidence of exacerbation or onset of new TM.

If rocuronium had to be used unavailability of sugammadex would be the limitation of this case report. Case of transverse myelitis is challenging is challenging for anesthesiologist due to varied response to muscle relaxants, higher incidence of autonomic dysreflexia. Considering all these possibilities adequate precautions and preparations were done. TOF monitoring for assessing adequacy of muscle relaxation, BIS for assessment of depth of anesthesia were used. As anticipated increased sensitivity to muscle relaxants was not observed which can be practiced in patients with TM. Safe anesthetic practice can be achieved in the case of 
transverse myelitis without exacerbating the neurological sequelae.

\section{CONCLUSION}

Perioperative management of patient with TM is challenging and poses unpredictable effects of anesthetic management. Thorough preoperative evaluation, adequate preparation, judicious use of anesthetic agents and close monitoring of vitals and neuromuscular function enables successful management of patient with transverse myelitis.

\section{FIGURE LEGENDS}

Figure 1

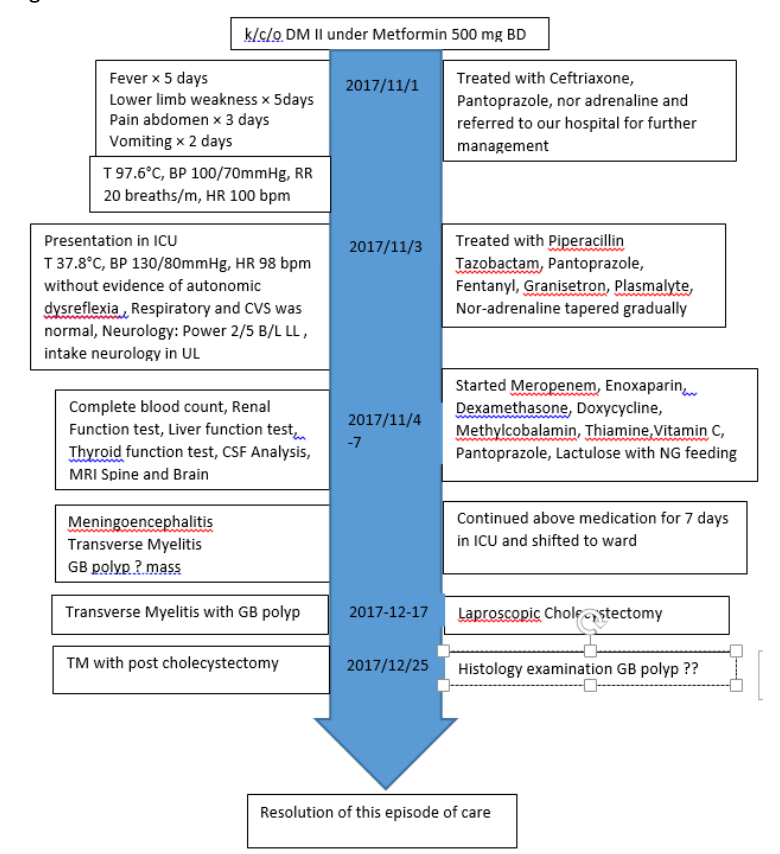

Figure 2

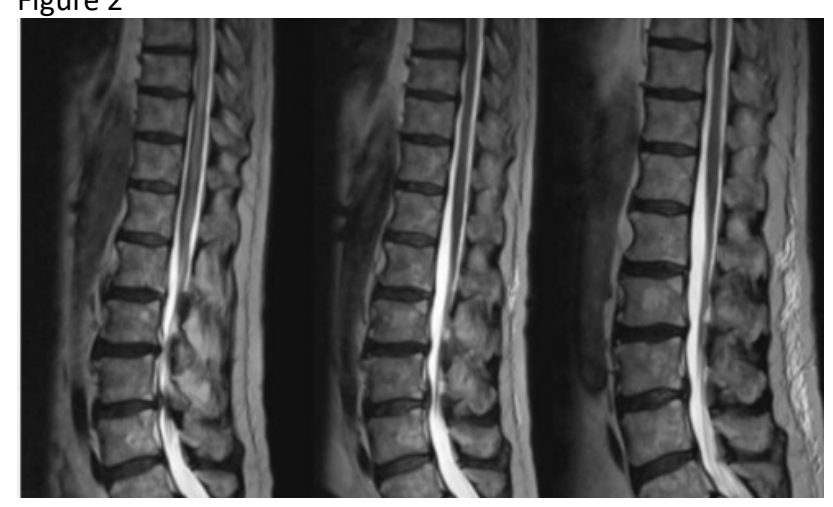

Figure 3

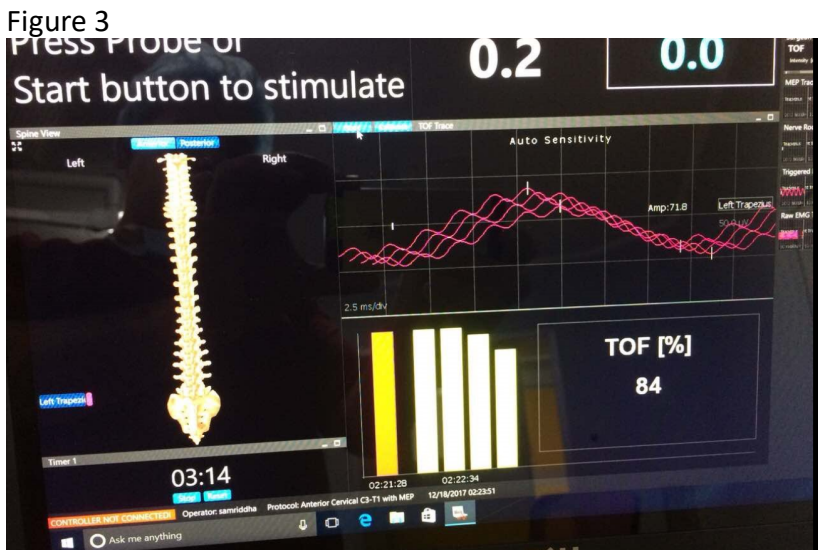

ABBREVIATIONS

BIS

$\mathrm{CO} 2$

CSEA

CSF

L2

$\llcorner 5$

MAC

MRI

SCI

TM

TOF

Bispectral index

Carbon dioxide

Combined spinal epidural analgesia

Cerebrospinal fluid

Second lumbar vertebra

Fifth lumbar vertebra

minimum alveolar concentration

Magnetic resonance imaging

spinal cord infarction

Transverse myelitis

Train-of-four

\section{Competing interests}

No authors have any financial and non-financial competing interests.

\section{Funding details}

The authors received no funding for the reports from any individual/institution.

\section{Authors' Contributions}

Dr. Achyut Sharma: primary author, actively involved in the management of the case, and drafting, reviewing, editing, preparing, and final approval of the manuscript.

Dr. Shikshya Khatiwoda, Dr. Kishor Khanal, Dr. Apurb Sharma, Dr. Prajjwal Raj Bhattarai: involved directly in patient management, reviewing, and final approval of the manuscript.

\section{ACKNOWLEDGEMENTS}

Dr. Hemant Adhikari, MD

Dr. Diptesh Aryal, MD, FACC

\section{REFERENCES}

1. Weekes G, Hayes N, Bowen M. Reversal of prolonged rocuronium neuromuscular blockade with sugammadex in an obstetric patient with transverse myelitis. Int J Obstet Anesth. 2010;19(3):333-6. 
2. Wu C, Zhang $Y, X u$ J. A confounding coincidence: Epidural anesthesia and paraplegia due to intramedullary tuberculoma in a patient who underwent cholecystectomy. BMC Anesthesiol. 2014;14(1):5-8.

3. Kaur R, Banerjee N. Transverse Myelitis Following Spinal Anaesthesia - a Case Report. Indian J Anaesth. 2002;46(5):476-7.

4. Seok JH, Lim YH, Woo SH, Yon JH. Transverse myelitis following combined spinal-epidural anesthesia. Korean J Anesthesiol. 2012;63(5):473-4.

5. Christ, G. H., Bonanno, G., MAlkinson, R., Rubin S. Transient paralysis associated with epidural steroid injection. J Spinal Disord. 1997;10(5):441-4.

6. Walsh P, Grange C, Beale N. Anaesthetic management of an obstetric patient with idiopathic acute transverse myelitis. Int J Obstet Anesth. 2010;19(1):98-101.

7. Balakrishnan IM, Yadav N, Singh GP, Prabhakar H. Anaesthetic considerations in patients with transverse myelitis. South African J Anaesth Analg. 2013;19(6):323-4. 